\title{
THE STEFAN PROBLEM WITH ARBITRARY INITIAL AND BOUNDARY CONDITIONS*
}

\author{
BY \\ L. N. TAO \\ Illinois Institute of Technology
}

\begin{abstract}
The paper is concerned with the free boundary problem of a semi-infinite body with an arbitrarily prescribed initial condition and an arbitrarily prescribed boundary condition at its face. An analytically exact solution of the problem is established, which is expressed in terms of some functions and polynomials of the similarity variable $x / t^{1 / 2}$ and time $t$. Convergence of the series solution is considered and proved. Hence the solution also serves as an existence proof. Some special initial and boundary conditions are discussed, which include the Neumann problem and the one-phase problem as special cases.
\end{abstract}

1. Introduction. Diffusive processes with a change of phase of the materials occur in many scientific and engineering problems. They are known as Stefan problems or free boundary problems. Many examples of these problems can be given, e.g. the melting or freezing of an ice-water combination, the crystallization of a binary alloy or dissolution of a gas bubble in a liquid. To find the solutions to this class of problems has been the subject of investigations by many researchers. Because of the presence of a moving boundary between the two phases, the problem is nonlinear. Various mathematical methods and techniques have been used to study free boundary problems. They have been discussed and summarized in several books [1-5] and many survey papers [6-9].

Free boundary problems have been studied since the nineteenth century by Lamé and Clapeyron in 1831, Neumann in the 1860s and Stefan in 1889. However, the only known exact solutions are those of Neumann and some of their extensions and variations. These solutions are all expressible in a single similarity variable $x / t^{1 / 2}$. This is to say that the partial differential equations of the problem are reducible to a set of ordinary differential equations. In the case of classical free boundary problems, the reductions are possible only when the body is semi-infinite and the boundary and initial conditions are of certain special forms. Neumann considered a semi-infinite body of a constant initial temperature which is suddenly in contact with a different temperature at its face. The exact solutions of the temperature of both phases are then found in the form of the similarity variable $x / t^{1 / 2}$. And the solution of the interface location is proportional to $t^{1 / 2}$. A generalization of the problem to cover arbitrary initial and boundary conditions is nontrivial, since the reduction to ordinary differential equations is no longer possible. No exact solutions have yet been found for the Neumann problem with arbitrary initial and boundary conditions.

It is the purpose of this paper to study the Neumann problem with arbitrary initial and boundary conditions, i.e., the free boundary problem of a semi-infinite body with an

* Received November 20, 1977; revised version received February 3, 1978. 
arbitrarily prescribed initial temperature, subject to an arbitrarily prescribed temperature at its face. It is found that an analytically exact solution to the problem can be established. The temperature solutions of both phases are expressed in terms of time $t$ and some polynomials and functions of the similarity variable $x / t^{1 / 2}$.

In the next section we shall first discuss some polynomials and functions related to the error integrals which are needed in this study. They are then used in Sec. 3 to solve the above-mentioned problem. The analytically exact solutions of the temperature of both phases and the interface location are formally established in the form of infinite series. We then prove the convergence of these series in Sec. 4. The establishment of the convergence also serves as a constructive proof of the existence of solutions. We conclude the paper with consideration of some problems with special boundary and initial conditions. The Neumann problem and the so-called one-phase problems are included as special cases.

2. Mathematical preliminaries. Solutions of the one-dimensional diffusion equation

$$
\alpha\left(\partial^{2} T / \partial x^{2}\right)=(\partial T / \partial t)
$$

may be expressed in terms of time $t$ and functions of the error integral family. These functions are defined by

$$
\begin{gathered}
\operatorname{erf} \xi=\frac{2}{\sqrt{ } \pi} \int_{0}^{\xi} \exp \left(-x^{2}\right) d x, \\
\operatorname{erfc} \xi=1-\operatorname{erf} \xi=\frac{2}{\sqrt{ } \pi} \int_{\xi}^{\infty} \exp \left(-x^{2}\right) d x, \\
i^{n} \operatorname{erfc} \xi=\operatorname{erfc} \xi, \quad i^{r} \operatorname{erfc} \xi=\int_{\xi}^{\infty} i^{n-1} \operatorname{erfc} x d x, \\
\Phi_{0}(\xi)=\operatorname{erf} \xi, \quad \Phi_{n}(\xi)=d^{n}(\operatorname{erf} \xi) / d \xi^{n}
\end{gathered}
$$

where $\xi=x /(4 \alpha t)^{1 / 2}$. The mathematical properties [1, 10] and tabulated values $[11,12]$ of these functions are well known. We recall the following properties:

$$
\begin{gathered}
i \operatorname{erfc} \xi=\pi^{-1 / 2} \exp \left(-\xi^{2}\right)-\xi \operatorname{erfc} \xi ; \\
4 i^{2} \operatorname{erfc} \xi=\operatorname{erfc} \xi-2 \xi i \operatorname{erfc} \xi ; \\
2 n i^{n} \operatorname{erfc} \xi=i^{n-2} \operatorname{erfc} \xi-2 \xi i^{n-1} \operatorname{erfc} \xi \\
i^{n} \operatorname{erfc}(0)=\left[2^{n} \Gamma(n / 2+1)\right]^{-1} ; \\
\Phi_{n}(\xi)=\frac{2}{\pi^{1 / 2}} \frac{d^{n-1}}{d \xi^{n-1}}\left(\exp \left(-\xi^{2}\right)\right)=\frac{2}{\pi^{1 / 2}}(-1)^{n-1} \exp \left(-\xi^{2}\right) H_{n-1}(\xi)
\end{gathered}
$$

where $H_{n}(\xi)$ is the Hermite polynomial.

In this paper we intend to use these functions with negative arguments as well as those with positive arguments. Functions with a negative argument also satisfy the diffusion equation and the above recurrence formulas. They are related to those of a positive argument by

$$
\begin{gathered}
\operatorname{erfc}(-\xi)=1+\operatorname{erf} \xi=2-\operatorname{erfc} \xi \\
i \operatorname{erfc}(-\xi)=2 \xi+i \operatorname{erfc} \xi
\end{gathered}
$$




$$
\begin{aligned}
i^{n} \operatorname{erfc}(-\xi)+(-1)^{n} i^{n} \operatorname{erfc} \xi & =\sum_{m=0}^{n}\left[1+(-1)^{m}\right] i^{m} \operatorname{erfc}(0) \frac{\xi^{n-m}}{(n-m) !} \\
& =\sum_{0}^{|n / 2|} \frac{\xi^{n-2 m}}{2^{2 m-1} m !(n-2 m) !} .
\end{aligned}
$$

The first two identities can be established from their definitions. Using the recurrence formula in (2.1), we may establish the third identity by mathematical induction.

For convenience, we introduce the following functions:

$$
\begin{gathered}
E_{n}(\xi)=\frac{1}{2}\left[i^{n} \operatorname{erfc}(-\xi)+i^{n} \operatorname{erfc} \xi\right], \\
F_{n}(\xi)=\frac{1}{2}\left[i^{n} \operatorname{erfc}(-\xi)-i^{n} \operatorname{erfc} \xi\right], \\
G_{n}(\xi)=\frac{1}{2}\left[i^{n} \operatorname{erfc}(-\xi)+(-1)^{n} i^{n} \operatorname{erfc} \xi\right] .
\end{gathered}
$$

Clearly,

$$
E_{2 n}(\xi)=G_{2 n}(\xi), \quad F_{2 n+1}(\xi)=G_{2 n+1}(\xi)
$$

and $G_{n}(\xi)$ is a polynomial of order $n$ and satisfies

$$
2 n G_{n}(\xi)=2 \xi G_{n-1}(\xi)+G_{n-2}(\xi) .
$$

Their integral representations may be given by

$$
\begin{gathered}
{\left[\begin{array}{l}
E_{n}(\xi) \\
F_{n}(\xi)
\end{array}\right]=\int_{0}^{\infty}[k(x-y, t) \pm k(x+y, t)]\left(\frac{y}{(4 \alpha t)^{1 / 2}}\right)^{n} \frac{d y}{n !},} \\
G_{n}(\xi)=\int_{-\infty}^{\infty} K(x-y, t)\left(\frac{y}{(4 \alpha t)^{1 / 2}}\right)^{n} \frac{d y}{n !}
\end{gathered}
$$

where

$$
K(x, t)=\frac{1}{(4 \pi \alpha t)^{1 / 2}} \exp \left[-x^{2} / 4 \alpha t\right]
$$

To verify the integral representation of $G_{n}(\xi)$, we integrate the expression and obtain

$$
\begin{gathered}
G_{0}=1, \quad G_{1}=\xi, \\
2 n G_{n}(\xi)=2 \xi G_{n-1}(\xi)+G_{n-2}(\xi) .
\end{gathered}
$$

This completes the verification. We may verify the other two integral representations similarly.

It may be noted that $E_{n}(\xi), F_{n}(\xi), G_{n}(\xi)$ and $i^{n}$ erfc $(-\xi)$ are undefined at $t=0$, but the product with $(4 \alpha t)^{n / 2}$ has a definite limit, e.g.,

$$
\left.(4 \alpha t)^{n / 2} G_{n}(\xi)\right|_{o}=\left.\frac{1}{2}(4 \alpha t)^{n / 2} i^{n} \operatorname{erfc}(-\xi)\right|_{o}=x^{n} / n ! .
$$

For later reference, we list some of the properties and values of these functions which are needed in this paper:

$$
\begin{aligned}
d^{m} G_{n}(\xi) / d \xi^{m} & =G_{n-m}(\xi) \quad \text { when } m \leq n, \\
= & 1 \quad m=n, \\
= & 0 \quad m>n, \\
d E_{n}(\xi) / d \xi=F_{n-1}(\xi), & d F_{n}(\xi) / d \xi=E_{n-1}(\xi),
\end{aligned}
$$




$$
\begin{gathered}
F_{n}(0)=G_{2 n+1}(0)=\Phi_{2 n}(0)=0, \\
E_{2 n}(0)=G_{2 n}(0)=1 / 2^{2 n} n ! \\
E_{2 n+1}(0)=1 /\left[2^{2 n+1} \Gamma\left(n+\frac{3}{2}\right)\right], \\
\Phi_{2 n+1}(0)=\frac{2}{\sqrt{\pi}} H_{2 n}(0)=(-1)^{n} \frac{2}{\sqrt{\pi}} \frac{(2 n) !}{n !} .
\end{gathered}
$$

3. Analytical solutions. Consider a material of constant properties in a semi-infinite region, $x>0$, with an initial temperature $V(x)$, which is suddenly in contact with a different temperature $U(t)$ at $x=0$. A new phase of the material starts to appear due to this sudden change of temperature at the boundary $x=0$. Let us denote these two phases, new and original, by subscripts I and II, respectively. Then the complete set of equations for this free boundary problem is

$$
\begin{gathered}
\alpha_{\mathrm{I}}\left(\partial^{2} T_{\mathrm{I}} / \partial x^{2}\right)=\partial T_{\mathrm{I}} / \partial t \quad 0<x<s(t), \\
\alpha_{\mathrm{II}}\left(\partial^{2} T_{\mathrm{II}} / \partial x^{2}\right)=\partial T_{\mathrm{II}} / \partial t \quad s(t)<x<\infty, \\
T_{\mathrm{I}}(0, t)=U(t), \quad T_{\mathrm{II}}(x, 0)=V(x), \\
T_{\mathrm{I}}(s, t)=T_{\mathrm{II}}(s, t)=T_{f}, \\
\left.k_{\mathrm{I}} \frac{\partial T_{\mathrm{I}}}{\partial x}\right|_{s}-\left.k_{\mathrm{II}} \frac{\partial T_{\mathrm{II}}}{\partial x}\right|_{s}= \pm \rho l \frac{d s}{d t}, \quad s(0)=0 .
\end{gathered}
$$

where $s(t)$ is the position of the interface. The sign in the heat balance equation depends upon whether there is a liberation or absorption of heat during the phase change. The other symbols have their usual meaning.

Let us consider specifically the solidification problem. Then the original phase is a liquid with an intial temperature $V(x)$ above or equal to the freezing temperature $T_{f}$. Solidification will occur only when the boundary temperature $U(t)$ is below $T_{f}$. Also, we take $U(0)$ as the reference temperature; then $U(0)=0$. We assume that both $U(t)$ and $V(x)$ are analytic functions of their respective argument and that $V(x)$ is bounded and $V^{\prime}(x)$ is of the exponential order $O(\exp (-\gamma x))$ or $V^{(n)}(x)=0$ as $x \rightarrow \infty$. In other words, they are expressible in the form of power series,

$$
\begin{gathered}
U(t)=\sum_{1} u_{n} t^{n} / n !, \quad u_{n}=\left(d^{n} U / d t^{n}\right)_{0}, \\
V(x)=\sum_{0} v_{n}\left(x / \alpha_{\mathrm{II}}^{1 / 2}\right)^{n} / n !, \quad v_{n}=\alpha_{\mathrm{II}}^{n / 2}\left(d^{n} V / d x^{n}\right)_{0} .
\end{gathered}
$$

We may now write the temperature solutions of this free boundary problem in the form

$$
\begin{gathered}
T_{\mathrm{I}}=\sum_{1} u_{n}(4 t)^{n} G_{2 n}\left(\xi_{\mathrm{I}}\right)+\sum_{0} a_{n}(4 t)^{n / 2} F_{n}\left(\xi_{\mathrm{I}}\right), \\
T_{\mathrm{II}}=\sum_{0} v_{n}(4 t)^{n / 2} G_{n}\left(\xi_{\mathrm{II}}\right)+\sum_{0} b_{n}(4 t)^{n / 2} i^{n} \operatorname{erfc} \xi_{\mathrm{II}},
\end{gathered}
$$

where $\xi_{i}=x /\left(4 \alpha_{i} t\right)^{1 / 2}$. It may be readily seen that every term in these two equations is a solution of their respective diffusion equation. Also, $T_{\mathrm{I}}$ satisfies the boundary condition at $x=0$ 


$$
T_{1}(0, t)=\sum_{1} u_{n}(4 t)^{n} G_{2 n}(0)+\sum_{0} a_{n}(4 t)^{n / 2} F_{n}(0)=U(t),
$$

and $T_{\mathrm{II}}$ satisfies the initial condition

$$
T_{\text {II }}(x, 0)=\left.\sum_{0} v_{n}(4 t)^{n / 2} G_{n}\left(\xi_{\text {II }}\right)\right|_{0}+\sum_{0} b_{n}(4 t)^{n / 2} i^{n} \operatorname{erfc}(\infty)=V(x) .
$$

Here we have used (2.5) and (2.6).

To complete the solution, we need to find the coefficients $a_{n}, b_{n}$ and the function $s(t)$ to satisfy the interface conditions:

$$
T_{\mathrm{I}}(s, t)=T_{\mathrm{II}}(s, t)=T_{f},\left.k_{\mathrm{I}} \frac{\partial T_{\mathrm{I}}}{\partial x}\right|_{s}-\left.k_{\mathrm{II}} \frac{\partial T_{\mathrm{II}}}{\partial x}\right|_{s}=\rho l \frac{d s}{d t} .
$$

Let us now write

$$
s(t)=2 \alpha_{1}^{1 / 2} t^{\nu} \chi(t)
$$

where $\nu>0$ and $\chi(t)$ is a function of $t$ with $\chi(0) \neq 0$. Substitution of this equation in (3.4) and evaluation at $\mathrm{t}=0$ yield $\nu=\frac{1}{2}$. Therefore

$$
s(t)=\left(4 \alpha_{1} t\right)^{1 / 2} \chi(t) .
$$

The usual approach for determining the coefficients would have been to express $\chi(t)$ in a power series of $t$ and equate the powers of $t$. A series of $\chi(t)$ in integer power of $t$, however, will not generally be able to satisfy all three interface conditions. A discussion on this point will be given later. It is necessary to express $\chi(t)$ as a power series of $t^{1 / 2}$. Let us write

$$
\begin{gathered}
s(t)=\left(4 \alpha_{\mathrm{I}}\right)^{1 / 2} \sum_{0} c_{n} \tau^{n+1}, \\
\eta_{\mathrm{I}}=s /\left(4 \alpha_{\mathrm{I}} t\right)^{1 / 2}=\sum_{0} c_{n} \tau^{n}, \quad \eta_{\mathrm{II}}=s /\left(4 \alpha_{\mathrm{II}} t\right)^{1 / 2}=\omega \sum_{0} c_{n} \tau^{n}
\end{gathered}
$$

where $\tau=t^{1 / 2}$ and $\omega=\left(\alpha_{1} / \alpha_{I I}\right)^{1 / 2}$. The coefficients $a_{n}, b_{n}$, and $c_{n}$ can now be determined by matching the powers of $\tau$, after all functions have been expanded in power series of $\tau$. However, we prefer to differentiate the interface equations successively with respect to $\tau$ and evaluate them at $\tau=0$. Also, to circumvent terms of negative powers of $\tau$ in the heatbalance equation, we differentiate this equation after it is multiplied by $\tau$. This is permissible since these equations are valid for all time. Differentiations yield

$$
\begin{gathered}
\left.T_{\mathrm{I}}(s, \tau)\right|_{0}=\left.T_{\mathrm{II}}(s, \tau)\right|_{0}=T_{f}, \\
\left.D_{\tau}^{N} T_{\mathrm{I}}(s, \tau)\right|_{0}=\left.D_{\tau}^{N} T_{\mathrm{II}}(s, \tau)\right|_{0}=0, \quad N=1,2, \cdots, \\
k_{\mathrm{I}} D_{\tau}^{N}\left(\left.\tau \frac{\partial T_{\mathrm{I}}}{\partial x}\right|_{s}\right)_{0}-k_{\mathrm{II}} D_{\tau}^{N}\left(\left.\tau \frac{\partial T_{\mathrm{II}}}{\partial x}\right|_{s}\right)_{0}=\alpha_{\mathrm{I}}^{1 / 2} \rho l(N+1) ! c_{N}, \quad N=0,1,2, \cdots .
\end{gathered}
$$

At $N=0$, we obtain

$$
a_{0}=T_{f} / \operatorname{erf}\left(c_{0}\right), \quad b_{0}=\left(T_{f}-v_{0}\right) / \operatorname{erfc}\left(\omega c_{0}\right)
$$

and $c_{0}$ satisfies

$$
\alpha_{0} \Phi_{1}\left(c_{0}\right)+b_{0} \omega\left(k_{\mathrm{II}} / k_{\mathrm{I}}\right) \Phi_{1}\left(\omega c_{0}\right)=\left(2 \rho l \alpha_{\mathrm{I}} / k_{\mathrm{I}}\right) c_{0} .
$$

To find the other coefficients, we first consider 


$$
\begin{aligned}
\left.D_{\tau}^{N}(2 \tau)^{n} G_{n}\left(\eta_{\mathrm{II}}\right)\right|_{0} & =\left.2^{n} \sum_{0}^{N}\left(\begin{array}{c}
N \\
m
\end{array}\right) \frac{n !}{(n-N+m) !} \tau^{n-N+m} D_{\tau}^{m} G_{n}\left(\eta_{\mathrm{II}}\right)\right|_{0} \\
& =\left.2^{n} \frac{N !}{(N-n) !} D_{\tau}^{N-n} G_{n}\left(\eta_{\mathrm{II}}\right)\right|_{0} .
\end{aligned}
$$

In order to evaluate $\left.D_{\tau}{ }^{m} G_{n}\left(\eta_{11}\right)\right|_{0}$, we use Faa de Bruno's formula for derivatives of a function of function $[13,14]$. Let $f=f(x)$ and $x=x(t)$; then

$$
\left.D^{m} f\right|_{t=0}=\left.\sum_{r=0}^{m} \frac{d^{r} f}{d x^{r}}\right|_{0} \sum_{\alpha_{i}} \frac{m !}{\alpha_{1} ! \alpha_{2} ! \cdots \alpha_{m} !}\left(D x_{0}\right)^{\alpha}\left(D^{2} x_{0} / 2 !\right)^{\alpha_{2}} \cdots\left(D^{m} x_{0} / m !\right)^{\alpha_{m}}
$$

where the second summation $\sum_{\alpha_{i}}$ is the sum extended to all values of $\alpha_{i}=0,1,2, \cdots$ such that

$$
\begin{gathered}
\alpha_{1}+\alpha_{2}+\cdots+\alpha_{m}=r, \\
\alpha_{1}+2 \alpha_{2}+\cdots+m \alpha_{m}=m .
\end{gathered}
$$

Using this formula, we find

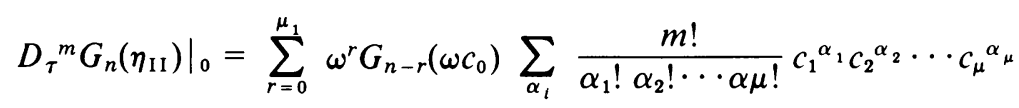

where $\mu_{1}=\min (m, n)$. Therefore

$$
\begin{gathered}
\left.D_{\tau}{ }^{N}(2 \tau)^{n} G_{n}\left(\eta_{1 \mathrm{II}}\right)\right|_{0}=N ! A_{n}{ }^{N}(\omega), \\
A_{n}{ }^{N}(\omega)=2^{n} \sum_{r=0}^{\mu_{\mu}} \omega^{r} G_{n-r}\left(\omega c_{0}\right) \sum_{\alpha_{i}} \frac{1}{\alpha_{1} ! \alpha_{2} ! \cdots \alpha_{\mu} !} c_{1}{ }^{\alpha} c_{2}{ }^{\alpha}{ }_{2} \cdots c_{\mu}{ }^{\alpha_{\mu}}, \\
\mu_{1}=\min (N-n, n), \alpha_{1}+\alpha_{2}+\cdots+\alpha_{\mu}=r, \alpha_{1}+2 \alpha_{2}+\cdots+\mu \alpha_{\mu}=N-n .
\end{gathered}
$$

Similar to the above derivation, we also have

$$
\left.D_{\tau}^{N}(2 \tau)^{n} i^{n} \operatorname{erfc}\left(\eta_{11}\right)\right|_{0}=N ! B_{n}^{N}(\omega)
$$

where

$$
B_{n}{ }^{N}(\omega)=2^{n} \sum_{r=0}^{N-n}(-\omega)^{r} i^{n-r} \operatorname{erfc}\left(\omega c_{0}\right) \sum_{\alpha_{i}} \frac{1}{\alpha_{1} ! \alpha_{2} ! \cdots \alpha_{\mu} !} c_{1}^{\alpha{ }}{c_{2}}^{\alpha_{2}} \cdots c_{\mu}{ }^{\alpha_{\mu}} .
$$

Here we have, for convenience, used the notation

$$
i^{-m} \operatorname{erfc} \xi=(-1)^{m+1} \Phi_{m}(\xi), \quad m>0 .
$$

Also, we have

$$
\begin{gathered}
D_{\tau}^{N}\left[2^{n} \tau^{n+1} G_{n}\left(\eta_{\mathrm{II}}\right)\right]_{0}=N ! P_{n}^{N}(\omega), \\
D_{\tau}^{N}\left[2^{n} \tau^{n+1} i^{n} \operatorname{erfc}\left(\eta_{\mathrm{II}}\right)\right]_{0}=N ! Q_{n}^{N}(\omega),
\end{gathered}
$$

where

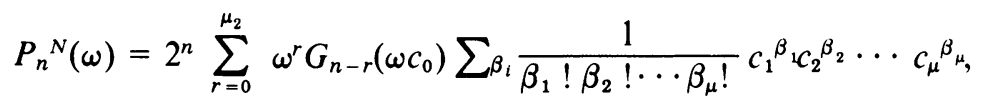




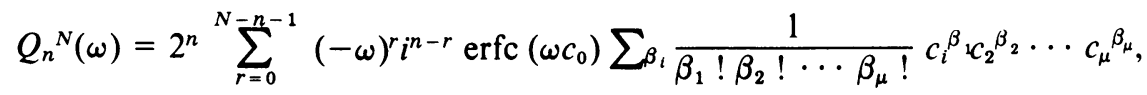

$$
\begin{aligned}
& \mu_{2}=\min (N-n-1, n)
\end{aligned}
$$

subject to

$$
\begin{gathered}
\beta_{1}+\beta_{2}+\cdots+\beta_{\mu}=r \\
\beta_{1}+2 \beta_{2}+\cdots+\mu \beta_{\mu}=N-n-1 .
\end{gathered}
$$

The interface conditions (3.6) can now be written as

$$
\begin{gathered}
\sum_{1}^{|N / 2|} u_{n} A_{2 n}{ }^{N}(1)+\sum_{0}^{N} a_{n} A_{n}{ }^{N}(1)-\sum_{0}^{|N / 3|} a_{2 n} B_{2 n}{ }^{N}(1)=0, \\
\sum_{1}^{N} v_{n} A_{n}{ }^{N}(\omega)+\sum_{0}^{N} b_{n} B_{n}{ }^{N}(\omega)=0, \\
\sum_{1}^{|N / 2|} u_{n} P_{2 n-1}{ }^{N}(1)+\sum_{0}^{N} a_{n} P_{n-1}{ }^{N}(1)+\sum_{0}^{|N / 2|} a_{2 n} Q_{2 n-1}{ }^{N}(1) \\
-\omega\left(k_{\mathrm{II}} / k_{\mathrm{I}}\right)\left[\sum_{1}^{N} v_{n} P_{n-1}{ }^{N}(\omega)-\sum_{0}^{N} b_{n} Q_{n-1}{ }^{N}(\omega)\right] \\
=\left(\rho l \alpha_{\mathrm{I}} / k_{\mathrm{I}}\right)(N+1) c_{N} .
\end{gathered}
$$

From this set of algebraic equations, we may determine, starting from $N=1$ step by step, the coefficients $a_{n}, b_{n}$ and $c_{n}$.

4. Existence and convergence. We have formally established in the preceding section the solutions of the free boundary problems. It remains to prove convergence of the series. This will not only complete the determination of the solutions, but also serve as a constructive type of proof of the existence of solutions. To this end, let us utilize an approach analogous to that used by Widder [15].

Let $s_{0}$ be the position of the interface at time $t_{0}$. Then the series

$$
\sum a_{n}\left(4 t_{0}\right)^{n / 2} F_{n}\left(\xi_{0}\right), \quad \xi_{0}=s_{0} /\left(4 \alpha t_{0}\right)^{1 / 2}
$$

converges, since $a_{n}$ are determined by the interface condition. Also, we observe that when $\xi>0$ both $i^{n} \operatorname{erfc}(-\xi)$ and $i^{n} \operatorname{erfc} \xi$ are positive. In addition, the former is monotonically increasing and the latter monotonically decreasing. This shows

$$
F_{n}(\xi) \leq F_{n}\left(\xi_{0}\right), \quad 0<\xi<\xi_{0} .
$$

Hence, $a_{n}$ is at most of the following order:

$$
a_{n}=0\left\{\left[2^{n} t_{0}{ }^{n / 2} F_{n}\left(\xi_{0}\right)\right]^{-1}\right\} .
$$

Using (4.2) and (4.3), we have

$$
\left|\sum a_{n}(4 t)^{n / 2} F_{n}(\xi)\right| \leq M_{1} \sum\left(\frac{t}{t_{0}}\right)^{n / 2} \frac{F_{n}(\xi)}{F_{n}\left(\xi_{0}\right)} \leq M_{1} \sum\left(\frac{t}{t_{0}}\right)^{n / 2}
$$

where $M_{1}$ is a constant independent of $n$. This is a geometric series. It implies that the given series converges, and hence $T_{\mathrm{I}}$ is bounded for all $\xi<\xi_{0}$ and $t<t_{0}$. 
Similarly, we have

$$
\begin{aligned}
i^{n} \operatorname{erfc} \xi & \leq i^{n} \operatorname{erfc} \xi_{0}, \quad x>s_{0}, t<t_{0}, \\
b_{n} & =0\left[\left(2^{n} t_{0}{ }^{n / 2} i^{n} \operatorname{erfc} \xi_{0}\right)^{-1}\right] .
\end{aligned}
$$

Therefore

$$
\left|\sum b_{n}(4 t)^{n / 2} i^{n} \operatorname{erfc} \xi\right| \leq M_{2} \sum\left(\frac{t}{t_{0}}\right)^{n / 2} \frac{i^{n} \operatorname{erfc} \xi}{i^{n} \operatorname{erfc} \xi_{0}} \leq M_{2} \sum\left(\frac{t}{t_{0}}\right)^{n / 2} .
$$

This establishes the boundness of $T_{\mathrm{II}}$.

Now let us consider the interface position $s(t)$. The third interface condition assures us that

$$
\sum a_{n}\left(4 t_{0}\right)^{n / 2-1} E_{n-1}\left(\xi_{0}\right), \quad \sum b_{n}\left(4 t_{0}\right)^{n / 2-1} i^{n-1} \operatorname{erfc} \xi_{0}
$$

exist and converge. When $\xi>0$ both $E_{n}(\xi)$ and $G_{n}(\xi)$ are positive. In addition, $G_{n}(\xi)$ are monotonically increasing. Hence

$$
\begin{array}{r}
E_{2 n}(\xi)=G_{2 n}(\xi) \geq G_{2 n}(0)=1 / 2^{2 n} n !, \\
\frac{E_{2 n+1}(\xi)}{x} \geq \frac{G_{2 n+1}(\xi)}{x} \geq\left.\frac{G_{2 n+1}(\xi)}{x}\right|_{0}=\frac{G_{2 n}(0)}{(4 \alpha t)^{1 / 2}},
\end{array}
$$

or

$$
E_{2 n+1}(\xi) \geq G_{2 n+1}(\xi) \geq \xi / 2^{2 n} n !
$$

Using Stirling's formula

$$
n ! \sim(n / e)^{n}(2 \pi n)^{1 / 2}
$$

we obtain

$$
a_{2 n+1}=O\left[\left(\frac{n}{e}\right)^{n} \frac{(2 n)^{1 / 2}}{t_{0}{ }^{n}}\right], \quad a_{2 n+2}=O\left[\left(\frac{n}{e}\right)^{n} \frac{(2 n)^{1 / 2}}{t_{0}{ }^{n+1 / 2}}\right]
$$

or

$$
a_{n+1}=O\left[\left(\frac{n}{2 e}\right)^{n} \frac{\sqrt{ } n}{t_{0}{ }^{n / 2}}\right] .
$$

Furthermore, we wish to establish an upper bound of $E_{n}(\xi)$. From

$$
x^{x} \geq \exp (x-1), \quad x>0
$$

and

$$
x=n /\left(2 c y^{2}\right), \quad c>0 \text { and } y>0,
$$

we obtain

$$
y^{n} \leq\left(\frac{n}{2 c e}\right)^{n / 2} \exp \left(c y^{2}\right)
$$

Substituting inţo (2.4), we have

$$
E_{n}(\xi) \leq \frac{1}{n !}\left(\frac{n}{2 c e}\right)^{n / 2}\left(\frac{1}{4 \alpha t}\right)^{n / 2} \int_{-\infty}^{\infty} \exp \left[-\frac{(x-y)^{2}}{4 \alpha t}+c y^{2}\right] d y
$$


Direct integration yields

$$
E_{n}(\xi) \leq \frac{1}{n !}\left(\frac{n}{2 c e}\right)^{n / 2}\left(\frac{1}{4 \alpha t}\right)^{n / 2} \frac{\exp \left[c x^{2} /(1-4 \alpha c t)\right]}{(1-4 \alpha c t)^{1 / 2}}
$$

Choosing

$$
c=(1-\epsilon) / 4 \alpha t, \quad 0<\epsilon<1
$$

we have

$$
E_{n}(\xi) \leq \frac{1}{n !}\left(\frac{n}{2 e}\right)^{n / 2}\left(\frac{1}{1-\epsilon}\right)^{n / 2} \frac{1}{\epsilon^{1 / 2}} \exp \left[\left(\frac{1-\epsilon}{\epsilon}\right) \frac{x^{2}}{4 \alpha t}\right]
$$

Thus

$$
\left|\sum a_{n}(4 t)^{(n-1) / 2} E_{n-1}(\xi)\right| \leq M_{3} \sum\left[\frac{t}{(1-\epsilon) t_{0}}\right]^{n / 2}
$$

where $M_{3}$ is a constant independent of $n$. This is essentially a geometric series. With arbitrary $\epsilon>0$, we conclude that $\partial T_{\mathrm{I}} / \partial x$ is bounded for all $x<s_{0}$ and $t<t_{0}$.

Similarly, we have

$$
b_{n+1}=O\left[\left(2^{n} t_{0}{ }^{n / 2} i^{n} \operatorname{erfc} \xi_{0}\right)^{-1}\right]
$$

and

$$
\left|\sum b_{n}(4 t)^{(n-1) / 2} i^{n} \operatorname{erfc} \xi\right| \leq M_{4} \sum\left(\frac{t}{t_{0}}\right)^{n / 2} .
$$

So $\partial T_{\mathrm{II}} / \partial x$ is also bounded for $x>s_{0}$ and $t<t_{0}$. With both $\partial T_{\mathrm{I}} / \partial x$ and $\partial T_{\mathrm{II}} / \partial x$ bounded in their respective domain, we conclude that $s(t)$ is bounded for all $t<t_{0}$.

5. Special cases. In this paper we have established an analytically exact solution to the free boundary problem of a semi-infinite body with arbitrarily prescribed initial and boundary conditions. The problem is reduced to a set of algebraic equations for determination of the coefficients $a_{n}, b_{n}$ and $c_{n}$. We now intend to discuss two special cases where the general formulas can be simplified.

In the case of the Neumann problem, where both $U(t)$ and $V(x)$ are constant, we have

$$
U(t)=0, \quad V(x)=V_{0}
$$

and

$$
u_{n}=v_{n}=0 \quad n=1,2, \cdots .
$$

The interface conditions (3.12) are reduced to a set of homogeneous equations; we readily obtain

$$
a_{n}=b_{n}=c_{n}=0 \quad n=1,2, \cdots .
$$

Therefore, the solutions of the problem are

$$
\begin{gathered}
T_{\mathrm{I}}=T_{f} \operatorname{erf} \xi_{\mathrm{I}} / \operatorname{erf}\left(c_{0}\right), \\
T_{\mathrm{II}}=\left(T_{f}-V_{0}\right) \operatorname{erfc} \xi_{\mathrm{II}} / \operatorname{erfc}\left(\omega c_{0}\right)+V_{0}, \\
s=c_{0}\left(4 \alpha_{\mathrm{I}} t\right)^{1 / 2},
\end{gathered}
$$


where $c_{0}$ satisfies

$$
\frac{T_{f} \Phi_{1}\left(c_{0}\right)}{\operatorname{erf}\left(c_{0}\right)}+\omega \frac{T_{f}-V_{0}}{\operatorname{erfc}\left(\omega c_{0}\right)}\left(\frac{k_{\mathrm{II}}}{k_{\mathrm{I}}}\right) \Phi_{1}\left(\omega c_{0}\right)=\frac{2 \rho l \alpha_{\mathrm{I}}}{k_{\mathrm{I}}} c_{0} .
$$

This is the well-known Neumann solution.

Let us now consider the problem where the initial condition $V(x)$ is an even function of $x$, i.e., $v_{2 n+1}=0$. We then find from (3.12) that

$$
a_{2 n+1}=b_{2 n+1}=c_{2 n+1}=0 .
$$

This may be seen from the fact that the initial and boundary conditions are related to the solutions of $T_{\mathrm{I}}$ and $T_{\mathrm{II}}$ only in the form of

$$
\sum u_{n}(2 t)^{2 n} G_{2 n}\left(\xi_{\mathrm{I}}\right), \sum v_{2 n}(2 t)^{2 n} G_{2 n}\left(\xi_{\mathrm{II}}\right)
$$

Both of these two series at the interface are even functions of $\tau$, if $s / \tau$ is an even function. In view of the requirement that $T_{\mathrm{I}}=T_{\mathrm{II}}=T_{f}$ at the interface, only even powers of $\tau$ are admissible. Hence all terms containing odd powers of $\tau$ must drop out. Thus, the solutions can be rewritten as

$$
\begin{gathered}
T_{\mathrm{I}}=\sum_{1} u_{n}(4 \tau)^{n} G_{2 n}\left(\xi_{\mathrm{I}}\right)+\sum_{0} a_{2 n}(4 t)^{n} F_{2 n}\left(\xi_{\mathrm{I}}\right), \\
T_{\mathrm{II}}=\sum_{0} v_{2 n}(4 \tau)^{n} G_{2 n}\left(\xi_{\mathrm{II}}\right)+\sum_{0} b_{2 n}(4 t)^{n} i^{2 n} \operatorname{erfc} \xi_{\mathrm{II}}, \\
s=\left(4 \alpha_{\mathrm{I}} t\right)^{1 / 2} \sum_{0} c_{2 n} t^{n} .
\end{gathered}
$$

The interface conditions become

$$
\begin{gathered}
\sum_{1}^{N} u_{n} A_{2 n}{ }^{2 N}(1)+\sum_{0}^{N} a_{2 n}\left[A_{2 n}{ }^{2 N}(1)-B_{2 n}{ }^{2 N}(1)\right]=0 \\
\sum_{1}^{N} v_{2 n} A_{2 n}{ }^{2 N}(\omega)+\sum_{0}^{N} b_{2 n} B_{2 n}{ }^{2 N}(\omega)=0 \\
\sum_{1}^{N} u_{n} P_{2 n-1}{ }^{2 N}(1)+\sum_{0}^{N} a_{2 n}\left[P_{2 n-1}{ }^{2 N}(1)+Q_{2 n-1}{ }^{2 N}(1)\right] \\
-\omega\left(k_{1 \mathrm{I}} / k_{\mathrm{I}}\right)\left[\sum_{1}^{N} v_{2 n} P_{2 n-1}{ }^{2 N}(\omega)-\sum_{0}^{N} b_{2 n} Q_{2 n-1}{ }^{2 N}(\omega)\right] \\
=\left(\rho l \alpha_{\mathrm{I}} / k_{\mathrm{I}}\right)(2 N+1) c_{2 N} .
\end{gathered}
$$

This discussion can be directly applied to the so-called one-phase problem where $V(x)=$ $T_{f}$. Then $T_{\mathrm{II}}$ is identically equal to $T_{f}$ and we may drop all $b_{n}(n=0,1,2, \cdots)$ from the above equations.

\section{REFERENCES}

[1] H. S. Carslaw and J. C. Jaeger, Conduction of heat in solids, 2nd ed., Oxford Univ. Press, 1959

[2] J. Crank, The mathematics of diffusion, 2nd ed., Oxford Univ. Press, 1975

[3] R. W. Ruddle, The solidification of castings, Inst. Metals, London, 1957 
[4] L. I. Rubinstein, The Stefan problem (English translation), Am. Math. Soc., 1971

[5] J. R. Ockendon and W. R. Hodgkins, Moving boundary problems in heat flow and diffusion, Oxford Univ. Press, 1975

[6] S. G. Bankoff, Heat conduction or diffusion with change of phase, Adv. Chem. Eng. 5, 75-150 (1964)

[7] J. C. Muehbauer and J. E. Sunderland, Heat conduction with freezing or melting, Appl. Mech. Rev. 18, 951959 (1965)

[8] W. A. Tiller, Principles of solidification, in Arts and sciences of growing crystals, ed. J. J. Gilman, Wiley, 1963

[9] B. A. Boley, Survey of recent developments in the fields of heat conduction in solids and thermo-elasticity, Nuclear Eng. \& Des. 18, 377-399 (1972)

[10] Bateman Manuscript Project, Higher transcendental functions, 3 vols., McGraw-Hill, 1955

[11] J. Kaye, A table of first eleven repeated integrals of the error function, J. Math. \& Phys. 34, 119-125 (1955)

[12] E. Jahnke and F. Emde, Tables of functions, Dover, 1945

[13] C. Jordan, Calculus of finite differences, Chelsea, 1965

[14] L. N. Tao, On the material time derivative of arbitrary order, Quart., Appl. Math., this issue

[15] D. V. Widder, The heat equation, Academic Press, 1975 